\title{
Multiple Left Ventricular Aneurysms in a Patient With Cardiac Sarcoidosis
}

\author{
Yasuko Yamaura, Nozomi Watanabe, Takashi Akasaka, Takahiro Kawamoto \\ and Kiyoshi Yoshida \\ Department of Cardiology, Kawasaki Medical School, Kurashiki, Japan
}

\begin{abstract}
A 30 year old man was admitted because of electrocardiographic abnormalities. Echocardiography revealed left ventricular aneurysms at the apex and the mid-inferior wall. The histological diagnosis by endomyocardial biopsy was sarcoidosis. Treatment with prednisolone was done. This is a rare case of multiple left ventricular aneurysms due to sarcoidosis, which was detected early by echocardiography.

(J Echocardiogr 2004; 2: 105-106)
\end{abstract}

Key words: cardiac sarcoidosis, echocardiography

A 30 year old man was referred to the cardiology department because of abnormalities of electrocardiogram (ECG). Although he had no cardiac symptom, his ECG demonstrated atrioventricular dissociation and intraventricular conduction disturbance. His physical examination, including ophthalmic and dermatic examination, revealed no significant abnormalities. His chest X-ray and laboratory examination indicated no abnormal findings excluding mildly elevated serum angitensin-converting enzyme (ACE) and serum lysozyme values. Echocardiography revealed aneurysmal change of the left ventricular wall at the mid-inferior segment (Figure 1A), and a small left ventricular aneurysm at the apex (Figure 1B). Single photon emission computed tomography (SPECT) with Thallium-201 (Tl-201) and Gallium-67 citrate (Ga-67) showed abnormal Ga-67 uptake and constant Tl-201 defects in the same segments where echocardiographic abnormalities were present. Coronary angiography demonstrated normal coronary arteries. Histological examination of the endomyocardial biopsy showed

Received November 1, 2004; revision received December 10, 2004; accepted December 15, 2004

Address for correspondence: Yasuko Yamaura, MD

Division of Cardiology, Hyogo Health Service Association,

4-4-20 Mikage-Honmachi, Higashinada-ku, Kobe 658-0046, Japan.

Telephone: +81-78-856-7226

Fax: +81-78-856-7220

E-mail: yasuko-yamaura@mvj.biglobe.ne.jp

(C) 2003 Journal of Echocardiography noncaseating granuloma with epithelioid cells (Figure 2). Then, a diagnosis of cardiac sarcoidosis was made, and treatment with prednisolone was started. After 2 weeks of steroid therapy, the patient's ECG returned to sinus rhythm and the patient has been doing well without cardiac symptoms.

The clinical diagnosis of cardiac sarcoidosis is difficult. However, in Japan, the majority of sarcoidosisrelated deaths are due to cardiac sarcoidosis [1, 2]. This is a rare case of multiple left ventricular aneurysms in a patient with sarcoidosis. To our knowledge, there was only one previous report of multiple cardiac aneurysms due to sarcoidosis, which showed aneurysms at the ventricular septum and the right ventricle and was treated by surgical aneurysmectomy [3]. In our case, multiple left ventricular aneurysms were diagnosed at a small size, and steroid therapy was successfully done. Early diagnosis and treatment may be the most important factors in improving the prognosis of cardiac sarcoidosis [4], therefore, echocardiography should be carefully examined in a patient who is thought to have sarcoidosis.

\section{References}

1. Iwai K, Tachibana T, Hosoda Y, Matsui Y. Sarcoidosis autopsies in Japan. Frequency and trend in the last 28 years. Sarcoidosis 1988; 5: 60-5.

2. Iwai K, Sekiguti M, Hosoda Y, DeRemee RA, Tazelaar HD, Sharma OP, Maheshwari A, Noguchi TI. Racial dif- 

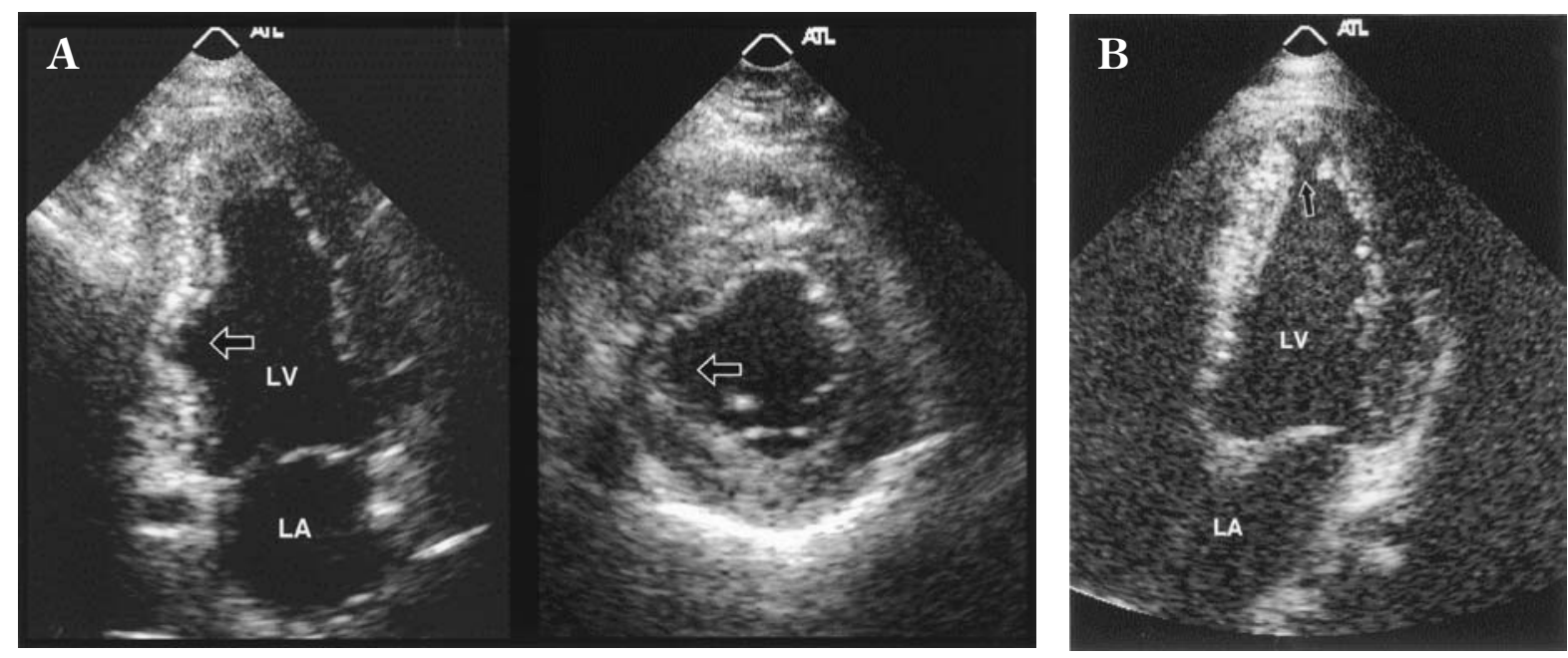

Fig. 1. A. Two-dimensional images of a left ventricular aneurysm at the mid inferior wall.

Echocardiography revealed aneurysmal change of the left ventricular wall (arrow) at the mid inferior segment. Left: apical two-chamber view. Right: short axis view.

B. Two-dimensional image of a left ventricular aneurysm at the apex.

Echocardiography demonstrated a small apical left ventricular aneurysm (arrow) in the apical four-chamber view. LV: left ventricle, LA: left atrium.

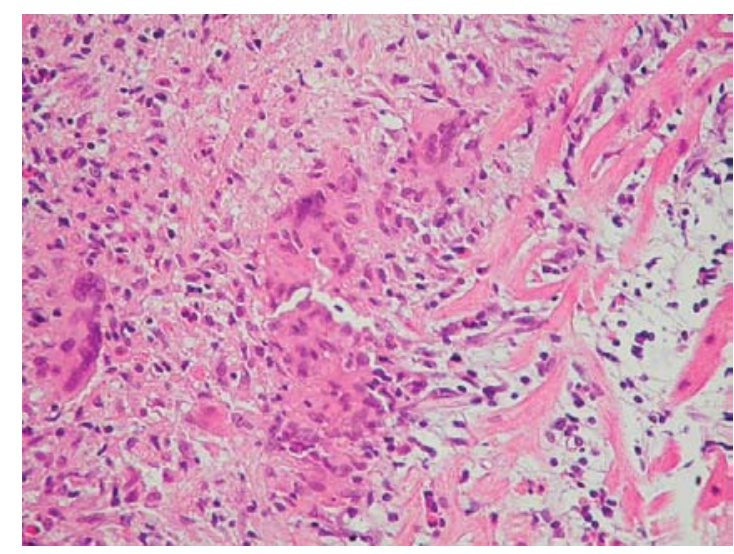

Fig. 2. The endomyocardial biopsy specimen demonstrates noncaseating granuloma with epithelioid cells. Hematoxilyn and eosin stain. ference in cardiac sarcoidosis incidence observed at autopsy. Sarcoidosis 1994; 11: 26-31.

3. Nielsen-Kudsk JE. Ventricular fibrillation and cardiac aneurysms caused by sarcoidosis. Ugeskr Laeger 1993; 155: 3299-301.

4. Silverman KJ, Hutchins GM, Burkley BH. Cardiac sarcoid: a clinicopathologic study of 84 unselected patients with systemic sarcoidosis. Circulation 1978; 58: 1204-11. 